\title{
PASEC 2014 et qualité des enseignants : une photographie pour un diagnostic
}

Hilaire Hounkpodote, Labass Lamine Diallo et Bassile Zavier Tankeu

\section{OpenEdition}

12 Journals

Édition électronique

URL : https://journals.openedition.org/ries/5885

DOI : $10.4000 /$ ries. 5885

ISSN : 2261-4265

Éditeur

France Education international

\section{Édition imprimée}

Date de publication : 1 septembre 2017

Pagination : 22-27

ISBN : 978-2-85420-615-9

ISSN : $1254-4590$

Référence électronique

Hilaire Hounkpodote, Labass Lamine Diallo et Bassile Zavier Tankeu, « PASEC 2014 et qualité des enseignants : une photographie pour un diagnostic », Revue internationale d'éducation de Sèvres [En ligne], 75 | septembre 2017, mis en ligne le 01 septembre 2019, consulté le 25 juin 2021. URL : http:// journals.openedition.org/ries/5885; DOI : https://doi.org/10.4000/ries.5885

Ce document a été généré automatiquement le 25 juin 2021.

(c) Tous droits réservés 


\title{
PASEC 2014 et qualité des enseignants : une photographie pour un diagnostic
}

\author{
Hilaire Hounkpodote, Labass Lamine Diallo et Bassile Zavier Tankeu
}

1 En Afrique subsaharienne francophone, la réflexion sur la question enseignante a longtemps été considérée comme secondaire, l'accent ayant été mis en priorité sur l'accès afin de satisfaire certains engagements pris dans le cadre des préoccupations internationales d'éducation. C'est le cas des politiques d'ajustement du secteur de l'éducation, qui ont impacté notamment la formation et le recrutement des enseignants (Bernard et al., 2004 ; Bourdon et Nkengné-Nkengné, 2007 ; Lauwerier et Akkari, 2015). Ces politiques ont contribué à la diversification concomitante des statuts professionnels (fonctionnaires, contractuels, communautaires...) et des profils de formation des enseignants.

2 Longtemps abordée sous l'angle du déficit quantitatif d'enseignant, la question enseignante s'installe progressivement à la faveur de la déclaration d'Incheon, au cœur des préoccupations nationales et internationales en termes de qualification des enseignants.

3 Dans cette dynamique, le rapport international et les rapports nationaux des dix pays participant à l'évaluation PASEC 2014 ont conduit à la production d'une analyse sur les profils de formation des enseignants des élèves testés en Afrique subsaharienne francophone. Ces rapports constituant le tremplin d'importantes réflexions sur les enseignants et les directeurs d'établissement campent aussi l'environnement de ces acteurs centraux des systèmes éducatifs, à travers une analyse du genre, de la formation académique, de la formation professionnelle et du regard sur leurs conditions matérielles de travail.

4 Les enseignants étant définis à travers l'objectif de développement durable $\mathrm{n}^{\circ} 4$ (ODD4), comme un moyen fondamental à renforcer pour l'atteinte d'une éducation de qualité pour tous dans le monde avant 2030, les données de l'évaluation PASEC 2014 proposent une analyse de cette cible comme préalable à cette action. Cet article présente un 
aspect de ce diagnostic, à savoir la situation de la formation académique et professionnelle des enseignants des classes primaires évaluées.

\section{Niveau académique des enseignants}

5 Le rapport international PASEC 2014 a analysé les divers niveaux de formation académique des enseignants des classes de début et de fin de scolarité primaire, dont les élèves ont été évalués. Les tableaux 1 et 2 présentent la répartition des élèves selon le niveau académique de leur enseignant (primaire, secondaire et universitaire).

Tableau 1. Répartition des élèves selon le niveau académique de l'enseignant - Début de scolarité

\begin{tabular}{|l|l|l|l|}
\hline & Niveau primaire & Niveau secondaire & Niveau universitaire \\
\hline Bénin & - & 80,3 & 19,7 \\
\hline Burkina Faso & 0,3 & 39,0 & 60,7 \\
\hline Burundi & 0,4 & 86,1 & 13,5 \\
\hline Cameroun & 1,6 & 81,3 & 17,2 \\
\hline Congo & - & 84,9 & 15,1 \\
\hline Côte d'Ivoire & - & 80,0 & 20,0 \\
\hline Niger & - & 93,3 & 6,7 \\
\hline Sénégal & - & 59,2 & 40,8 \\
\hline Tchad & - & 71,5 & 28,5 \\
\hline Togo & - & 90,6 & 9,4 \\
\hline
\end{tabular}

Tableau 2. Répartition des élèves selon le niveau académique de l'enseignant - Fin de scolarité

\begin{tabular}{|l|l|l|l|}
\hline & Niveau primaire & Niveau secondaire & Niveau universitaire \\
\hline Bénin & - & 76,8 & 23,2 \\
\hline Burkina Faso & 1,5 & 38,1 & 60,4 \\
\hline Burundi & 1,2 & 74,2 & 24,6 \\
\hline Cameroun & 0,0 & 72,2 & 27,8 \\
\hline Congo & 0,3 & 65,7 & 34,0 \\
\hline Côte d'Ivoire & 1,0 & 47,2 & 51,8 \\
\hline
\end{tabular}




\begin{tabular}{|l|l|l|l|}
\hline Niger & - & 70,5 & 29,5 \\
\hline Sénégal & - & 52,8 & 47,2 \\
\hline Tchad & - & 41,9 & 58,1 \\
\hline Togo & - & 71,5 & 28,5 \\
\hline
\end{tabular}

6 Premier constat sur le niveau académique des enseignants : en général, dans tous les pays évalués, la quasi-totalité des élèves en début et en fin du cycle primaire est encadrée par un enseignant dont le niveau académique est supérieur au primaire. La majorité des élèves a un enseignant de niveau secondaire, à l'exception du Burkina Faso ( $39,0 \%$ en début et $38,1 \%$ en fin de scolarité) et, en fin de scolarité, de la Côte d'Ivoire (47,2\%) et du Tchad (41,9\%). Dans le cas de ces pays, la majorité des élèves est encadrée par des enseignants ayant une formation universitaire.

7 À l'inverse, dans de nombreux pays, les pourcentages d'élèves encadrés par un enseignant ayant une formation universitaire sont bien plus faibles : moins de $25 \%$ au Bénin et au Burundi en fin de scolarité primaire, et moins de $20 \%$ dans six pays en début de scolarité (Bénin, Burundi, Cameroun, Congo, Niger et Togo).

Par ailleurs, dans tous les pays, le pourcentage d'élèves dont les enseignants ont un niveau d'enseignement supérieur, est plus élevé en fin de cycle qu'en début de cycle, à l'exception du Burkina Faso, où ce pourcentage est presque identique.

\section{Formation professionnelle initiale des enseignants}

Le PASEC 2014 a tenté d'apprécier le niveau de la formation professionnelle initiale des enseignants à travers la durée de cette formation, indépendamment de leur niveau académique, afin de faciliter la comparabilité, les types de formations spécifiques à l'enseignement pouvant varier entre pays.

Les tableaux 3 et 4 montrent la répartition des élèves selon la durée de la formation professionnelle initiale de l'enseignant, répartie en quatre catégories.

Tableau 3. Répartition des élèves selon la formation professionnelle de l'enseignant - Début de scolarité

\begin{tabular}{|l|l|l|l|l|}
\hline & Aucune formation professionnelle & Moins de six mois & Un an & Deux ans et plus \\
\hline Bénin & 15,6 & 4,9 & 10,5 & 69 \\
\hline Burkina Faso & 14,6 & 17,3 & 45,3 & 22,8 \\
\hline Burundi & 10,1 & 9 & 8,2 & 72,7 \\
\hline Cameroun & 20,7 & 2,4 & 17,6 & 59,3 \\
\hline Congo & 15,7 & 17,8 & 6,8 & 59,7 \\
\hline Côte d'Ivoire & 20,3 & 15,2 & 28,3 & 36,3 \\
\hline
\end{tabular}




\begin{tabular}{|l|l|l|l|l|}
\hline Niger & 23,3 & 21,2 & 28,7 & 26,8 \\
\hline Sénégal & 5,9 & 29,6 & 58 & 6,5 \\
\hline Tchad & 23,9 & 42,1 & 26,6 & 7,4 \\
\hline Togo & 67,2 & 23,8 & 7 & 2 \\
\hline Moyenne & 21,5 & 18,3 & 23,8 & 36,3 \\
\hline
\end{tabular}

Tableau 4. Répartition des élèves selon la formation professionnelle de l'enseignant - Fin de scolarité

\begin{tabular}{|l|l|l|l|l|}
\hline & Aucune formation professionnelle & Moins de six mois & Un an & Deux ans et plus \\
\hline Bénin & 4,2 & 18,4 & 28,6 & 48,8 \\
\hline Burkina Faso & 14,8 & 8,4 & 39,4 & 37,4 \\
\hline Burundi & 10,2 & 10,2 & 11,4 & 68,2 \\
\hline Cameroun & 8,6 & 2,7 & 35,3 & 53,4 \\
\hline Congo & 7,9 & 8,4 & 19,2 & 64,5 \\
\hline Côte d'Ivoire & 3,1 & 9,6 & 23,2 & 64,1 \\
\hline Niger & 4,1 & 12,8 & 38,5 & 44,7 \\
\hline Sénégal & 4,2 & 31,9 & 57,5 & 6,3 \\
\hline Tchad & 10,9 & 15,5 & 48,4 & 25,3 \\
\hline Togo & 34,3 & 48 & 10,5 & 7,2 \\
\hline Moyenne & 10,2 & 16,6 & 31 & 42,2 \\
\hline
\end{tabular}

11 L'évaluation montre qu'il existe dans tous les pays évalués une proportion non négligeable d'élèves dont l'enseignant n'a reçu aucune formation professionnelle initiale. La plupart des pays a en effet été confrontée à la nécessité d'une réponse urgente au défi de l'accès, par le recrutement important d'enseignants ne disposant souvent pas de formation pour cette profession. Dans certains cas, les enseignants ont reçu une formation pédagogique de quelques jours. Cette situation a le plus souvent débouché sur une différenciation des statuts et des conditions contractuelles.

En début du cycle, cette proportion atteint $21,5 \%$, en moyenne. Des variations sensibles autour de cette moyenne sont notables. Au Sénégal, le pourcentage d'élèves dont l'enseignant n'a aucune formation professionnelle initiale est comparativement faible $(5,9 \%)$. Cette proportion dépasse $20 \%$ au Cameroun $(20,7 \%)$, en Côte d'Ivoire $(20,3 \%)$, au Niger $(23,3 \%)$ et au Tchad (23,3\%), et atteint des proportions alarmantes au Togo 
(67,2 \%). En fin de cycle, la part d'élèves dont l'enseignant n'a aucune formation initiale est inférieure à $11 \%$, pour tous les pays sauf le Burundi $(14,8 \%)$ et le Togo $(34,3 \%)$.

La proportion d'élèves dont l'enseignant a bénéficié d'une formation initiale de moins de six mois varie de $2,4 \%$ au Cameroun à $42,1 \%$ au Tchad, en début de cycle. En fin de cycle, cette proportion se situe entre $2,7 \%$ au Cameroun et $48,0 \%$ au Togo. Ici encore, il est constaté que le début de scolarité primaire n'est pas prioritaire pour l'attribution d'enseignants formés.

Dans la plupart des pays, aussi bien en début qu'en fin de cycle, une grande majorité d'élèves ont un enseignant qui a suivi au moins un an de formation professionnelle, hormis au Togo, où seulement $17,7 \%$ d'élèves de $6 e$ année et $9,0 \%$ d'élèves de $2 e$ année ont un enseignant ayant reçu au moins un an de formation initiale.

En fin de cycle, le problème de l'absence de formation des enseignants ou de la faible durée de leur formation initiale est moins alarmant au Cameroun, au Congo, en Côte d'Ivoire et au Niger, comparativement à des pays comme le Togo, le Sénégal et Tchad.

L'analyse du profil professionnel initial des enseignants, mis en relation avec leur niveau académique et leur participation à des formations complémentaires, montre que l'absence de formation initiale du maître n'implique pas forcément une absence totale de formation pédagogique. À l'exception du Burundi, parmi les élèves ayant des enseignants sans formation professionnelle initiale, plus de $70 \%$ de ces élèves ont des enseignants ayant suivi une formation complémentaire en pédagogie. Il est également intéressant de constater que l'absence de formation initiale touche les enseignants de tous les niveaux académiques.

\section{Formation continue des enseignants}

17 La formation continue offre à l'enseignant l'opportunité de parfaire ses aptitudes professionnelles, de développer de nouvelles compétences pédagogiques, d'apprendre à mieux connaître et à appliquer les programmes scolaires. Dans des contextes de forte présence d'enseignants sans formation initiale professionnelle, elle se présente comme une alternative importante pour améliorer la qualité du système éducatif. Les instruments de collecte des données contextuelles du PASEC 2014 ont abordé la question de la formation continue des enseignants selon le nombre de jours de formation complémentaire reçue au cours des deux dernières années. Pour les besoins des analyses, le nombre de jours de formation a été scindé en cinq catégories (absence de formation continue, entre un et cinq jours de formation, entre six et dix jours de formation, entre onze et vingt jours de formation et plus de vingt jours de formation).

L'absence de formation continue des enseignants demeure une réalité, que ce soit en début ou en fin de scolarité primaire. Le pourcentage d'élèves est compris entre 2,2\% au Cameroun et $63,0 \%$ au Burundi en début de cycle, et entre 4,5 \% au Sénégal et $41,8 \%$ au Burundi en fin de cycle. Le Burundi est, pour les deux niveaux, le pays qui compte le plus d'élèves dont l'enseignant n'a reçu aucune formation continue.

Toutefois, les pourcentages d'élèves encadrés par un enseignant ayant reçu au moins quelques jours de formation complémentaire sont élevés, dans presque tous les pays, et particulièrement au Cameroun et au Sénégal (plus de $95 \%$ ). Parmi les enseignants ayant bénéficié de formation, la répartition selon le nombre de jours de formation 
montre, pour certains pays, une concentration dans la catégorie comprise entre un et cinq jours de formation.

Le PASEC 2014 a permis de réaliser une première photographie de constat comparatif sur les enseignants de dix pays d'Afrique subsaharienne francophone, qui met en évidence les insuffisances et diversités liées à la formation de la profession enseignante. Faute de place, nous ne pouvons mener ici l'analyse du lien entre le niveau académique et les différentes catégories de formation (professionnelle initiale et continue) des enseignants avec les performances des élèves, qui reste à conduire.

Le rapport des enseignants à la formation initiale spécifique à l'enseignement et à la formation continue dans des contextes scolaires complexes caractérisés, entre autres, par une insuffisance de ressources pédagogiques et des tailles de classe importantes constitue un véritable obstacle à la qualité de l'enseignement-apprentissage et à la construction d'une professionnalité enseignante.

L'hétérogénéité des niveaux de formation académique, des modes et des parcours de formation, la répartition inéquitable des profils de formation dans les systèmes éducatifs subsahariens francophones, la faiblesse du volume de formation professionnelle enseignante et le faible volume de temps consacré à la formation continue constituent un éventail de constats qui confortent l'ODD 4, point $\mathrm{C}$ : « d'ici à 2030, accroître considérablement le nombre d'enseignants qualifiés... ». Qui plus est, ce constat relatif à la formation des enseignants invite à aller plus loin dans la définition de ce qui est entendu et attendu à travers la «qualification des enseignants " dans l'ODD 4C. Un enseignant qualifié est-il celui qui présente les certifications attendues ou celui qui manifeste effectivement les compétences attendues pour l'enseignement? La détention de la certification pour l'enseignement n'étant pas un gage absolu de la manifestation des compétences d'enseignement, le PASEC s'est engagé, dans sa deuxième vague d'évaluation comparative internationale intitulée PASEC 2019, à évaluer les compétences des enseignants sur des disciplines fondamentales enseignées (mathématiques et langue d'enseignement) et sur le savoir didactique (maîtrise des objectifs d'apprentissage et typologie des erreurs).

\section{INDEX}

Mots-clés : évaluation, formation continue, formation des enseignants, formation initiale, profession d'enseignant, PASEC : programme d'analyse des systèmes éducatifs, ODD : objectifs de développement durable

Palabras claves : evaluación, educación recurrente, formación de docentes, formación inicial, docencia, PASEC: programa de análisis de los sistemas educativos, ODS: objetivos de desarrollo sostenible

Keywords : evaluation, recurrent education, teacher education, initial training, teaching profession, PASEC: Programme for the Analysis of the Educational Systems, SDGs: Sustainable Development Goals

Index géographique : Afrique subsaharienne 


\section{AUTEURS}

\section{HILAIRE HOUNKPODOTE}

Hilaire Hounkpodote est coordonnateur par intérim du Programme d'analyse des systèmes éducatifs (PASEC) de la Conférence des ministres de l'éducation des pays ayant le français en partage (Confemen). Il est ingénieur statisticien économiste, diplômé de l'École nationale de statistique et d'économie appliquée (ENSEA) d'Abidjan (Côte d'Ivoire). Courriel :

hhilaire@confemen.org

\section{LABASS LAMINE DIALLO}

Labass Lamine Diallo est docteur en sciences de l'éducation (Université de Bordeaux II) et a été enseignant-chercheur à l'université des lettres, langues et sciences humaines de Bamako (Mali). Il est actuellement conseiller technique à la Conférence des ministres de l'éducation des pays ayant le français en partage (Confemen). Courriel : ldiallo@confemen.org

\section{BASSILE ZAVIER TANKEU}

Bassile Zavier Tankeu est conseiller technique au Programme d'analyse des systèmes éducatifs de la Conférence des ministres de l'éducation des pays ayant le français en partage (Confemen). Il est ingénieur principal des travaux de la statistique, diplômé de l'Institut sous-régional de statistique et d'économie appliquée (ISSEA) de Yaoundé (Cameroun). 